\title{
DV-Hop Localization Scheme for WSN based on Artificial Bee Colony
}

\author{
Zhang Yibo \\ Chongqing College of Electronic Engineering, Chongqing, 401331, China \\ Email: 534878774@qq.com
}

Keywords: WSN; DV-Hop; Artificial Bee Colony; PSO

\begin{abstract}
In order to reduce the node localization error of DV-Hop algorithm in Wireless Sensor Network, an improved algorithm based on Hybrid Artificial Bee Colony algorithm is proposed. Combining the fast convergence speed characteristics of Particle Swarm Optimization(PSO) and strong searching capability of ABC, improved algorithm firstly estimating the distance between unknown nodes and anchor-nodes by DV-Hop algorithm. Secondly, the initial position of unknown nodes was calculated by PSO. Finally, the iterative numerical method with the initial values of estimated node locations was presented by ABC. It can be concluded that the improved algorithm has obviously better locating precision than PSO and DV-Hop.
\end{abstract}

\section{Introduction}

The existing localization algorithms in wireless sensor networks can be divided into two categories: ranging-based localization and non-ranging based localization. The former needs to obtain the distance information or angle information between nodes through the actual measurement method, and then uses the trilateral measurement method or maximum likelihood estimation method to calculate the location of nodes; the latter does not need actual measurement, only according to the network connection, node location is achieved by information such as degree. Ranging-based localization methods need to add additional hardware equipment and traffic, which increases energy consumption and costs; non-ranging localization methods need no additional hardware equipment, can reduce the hardware requirements of nodes, more suitable for the practical application of large-scale wireless sensor networks. Therefore, the non ranging location method has attracted more and more attention from scholars both at home and abroad. [3].

DV-Hop algorithm is one of the most concerned localization algorithms at present. Its advantage is that it is not affected by ranging error and has strong robustness, but it is only suitable for isotropic uniform network, and the positioning error is large. Artificial Bee Colony algorithm (ABC) is an optimization algorithm based on intelligent search behavior of bee colony, which was proposed by Karaboga of Ergis University in Turkey in 2005. At present, the research and application of ABC algorithm is still in the primary stage, but because of its few control parameters, easy to implement, simple calculation and other advantages, it has attracted more and more scholars' attention. Based on the analysis of the principle of the bee colony algorithm, this paper combines the bee colony algorithm with the particle swarm algorithm and applies it to the node of DV-Hop algorithm. Positioning stage, in order to improve the positioning accuracy of DV-Hop algorithm.

\section{Algorithm description}

\subsection{DV-Hop algorithm description}

DV-hop algorithm is proposed by Niculescu et al. Its localization process is divided into three stages:

1) Using a typical distance vector switching protocol, all nodes in the network can obtain the hop distance between themselves and anchor nodes, each anchor node calculates its own average hop distance according to formula (1): 


$$
\text { HopSize }_{i}=\sum_{j \neq i} \sqrt{\left(x_{i}-x_{j}\right)^{2}+\left(\mathrm{y}_{i}-y_{j}\right)^{2}} / \sum_{j \neq i} \text { hopS }_{i j}
$$

In formula (1), $\mathrm{j}$ is the other anchor node in the data table of anchor node $\mathrm{i}$, and hops $_{i j}$ is the hop count between anchor nodes $\mathrm{i}$ and $\mathrm{j}$.

2) Each anchor node broadcasts to the network according to the average hop distance computed by itself in the first stage, and each anchor node receives only the first average hop distance and discards all subsequent information.

3) According to the estimated distance computed in the previous stage, the unknown nodes use the trilateral or multilateral positioning method to calculate the estimated coordinates, so as to complete the positioning.

\subsection{Standard particle swarm optimization algorithm description}

Yuhui Shi and Eberhar proposed an improved particle swarm optimization algorithm, that is, adding an inertia factor $\mathrm{W}$ to the basic particle swarm optimization algorithm to adjust the impact of the last moment speed on the next moment speed, but also on the global search ability and local search ability to play a balancing role, the update of standard PSO algorithm can be described with formula (2) and formula (3):

$$
\begin{gathered}
V_{i}^{t+1}=w V_{i}^{t}+c_{1} r_{1}\left(P_{i}^{t}-X_{i}^{t}\right)+c_{2} r_{2}\left(P_{g}^{t}-X_{i}^{t}\right) \\
X_{i}^{t+1}=X_{i}^{t}+V_{i}^{t+1}
\end{gathered}
$$

In formula (2) and formula (3), $v$ denotes the velocity of the particle, $x$ denotes the unknown node of the particle, i denotes the first particle, $\mathrm{w}$ denotes the inertia factor, c1,c2 are positive acceleration factors, $\mathrm{r} 1$, r2 are uniformly distributed random numbers between $[0,1]$, p denotes the optimal position that a single individual has found in history, pg is the optimal position that the entire population has found, $t$ represents the T iteration.

\section{3 description of bee colony algorithm}

Firstly, the ABC algorithm generates an initial colony of bees with SN solutions (food sources), and each solution $\mathrm{Xi}$ is a d-Dimension vector. Then, the bees search all food sources in a circular fashion with the number of cycles $c(c=1,2, \ldots N)$. If the nectar quantity (fitness) of the food source (solution) is better than that of the previous one, the new food source position is used instead of the old one, otherwise the old food source position will remain unchanged. After all the lead bees had finished their search, they went back to the dance area to communicate the amount of nectar on the food source to the followers through a swing dance. The bee follows the information obtained according to the probability to select the food source. The more food is nectar, the greater the probability of being selected. Once the food source is selected, a neighborhood search is also carried out, and a better solution is retained. ABC algorithm is ultimately to find the optimal solution through such repeated search.

For ABC algorithm, the follower bee chooses the food source by observing the wagging dance of the leader bee to determine the yield of the food source, and according to the size of the yield to choose which food source to collect honey.

In the ABC algorithm, there is also a control parameter limit, which is used to record the number of times a solution is updated. Assuming that a solution does not improve after successive limit times cycles, indicating that the solution falls into local optimum, then the position will be abandoned, and the corresponding leader bee will also be turned into a scout bee.

\section{Improved algorithm}

Artificial bee colony algorithm is superior to particle swarm algorithm in both global and local search accuracy, but its convergence speed is slow. To compensate for this shortcoming, the global optimal value obtained by particle swarm algorithm is proposed as the initial solution of artificial 
bee colony algorithm in order to speed up the bee colony algorithm. The convergence speed and precision of the algorithm can be reduced, and the computation of the artificial bee colony algorithm can be reduced, so that the better solution can be obtained.

Based on this, an improved DV-Hop algorithm based on hybrid artificial bee colony algorithm is proposed in this paper. The main idea is to calculate the average hop distance and the distance between unknown nodes and anchor nodes by DV-Hop algorithm, and then obtain the global optimal solution by particle swarm optimization algorithm with fewer iterations, which is regarded as the initial value of the bee colony algorithm. Then the bee colony algorithm is used to optimize the unknown node coordinates to complete the final positioning. The main steps are as follows:

1) determine parameters: inertia factor $w$, iteration number $t$, population number $n$, cycle number $\mathrm{c}$ and control parameter limit

2) randomly producing populations of $\mathrm{N}$ particles.

3) update the particle velocity and position by using formula (2) and (3).

4) by calculating fitness, the global optimal solution PG is used as the initial solution of artificial bee colony algorithm.

5) calculate the probability value pi related to pg.

6) The follower bee chooses food source (solution) according to pi, carries on the neighborhood search to produce the new solution vi, calculates its fitness value.

7) if vi is better than pg replace pg with vi, or leave vi unchanged.

8) to determine whether the solution to be abandoned is returned to step $\mathrm{f}$ if it exists, generating a new solution instead of it.

9) record the best solution so far.

10) determine whether the loop termination condition is satisfied, such as satisfying the output best result, otherwise, step back to step f.

\section{Simulation experiment}

\section{1 simulation environment}

The experiments in this paper are carried out on the NS-2 platform, in order to verify the performance of the improved algorithm, the nodes are randomly arranged in the network area of $200 \mathrm{~m} \times 200 \mathrm{~m}$, the coordinates of unknown nodes and anchor nodes are randomly generated, and the communication radius of each node is $30 \mathrm{~m}$. Assuming the number of simulation times is $\mathrm{s}$, the number of anchor nodes is $\mathrm{m}$, the number of nodes is $\mathrm{n}$, the real coordinates of positioning nodes are (xt, yt), and the estimated coordinates are (xe, ye), the positioning error of a single node is ei, and the average positioning error of the whole network is enet, then the normalized average positioning error based on the statistics of s-tims simulation results is calculated as:

$$
e=\sum_{j=1}^{s} e_{n e t} / S R
$$

In order to objectively verify the positioning performance of the improved algorithm and facilitate the comparison of the traditional DV-Hop algorithm, the following settings are made in the simulation:

1) The network area, the total number of nodes, the total number of anchor nodes and the communication radius of nodes are the same in each simulation.

2) Statistical normalization positioning error is used as a measure index of algorithm accuracy.

\section{2 parameter setting}

Because wireless sensor network nodes are limited by volume and energy, in order to minimize the positioning error and energy consumption of the improved bee colony algorithm, the HABC algorithm is simulated by NS-2 simulation software in terms of the number of cycles and the final positioning performance. Let the nodes be randomly distributed in a square area of $200 \mathrm{~m}$. It is assumed that all anchor nodes are distributed in the first quadrant, the coordinates of anchor nodes 
are known, and the distance between anchor nodes and unknown nodes is known.

\section{3 simulation results analysis}

Through 1000 simulation experiments, the normalized positioning accuracy of the traditional DV-Hop algorithm and the proposed DV-Hop algorithm based on the HABC algorithm (HADV-Hop) are calculated, and the positioning performance of the two algorithms is analyzed and compared objectively. Fig. 1 shows the variation of the normalized average positioning error calculated by equation (4) when the ratio of anchor nodes varies from $5 \%$ to $40 \%$, the communication radius $\mathrm{R}=30 \mathrm{~m}$, and the total number of nodes is 180 .

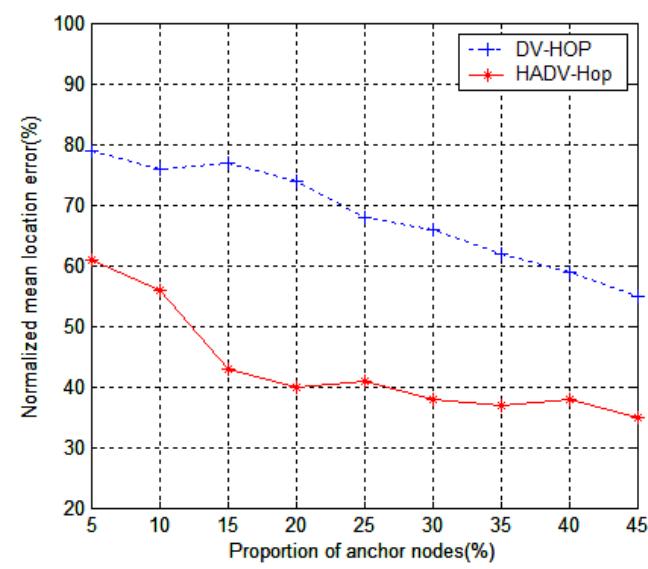

Fig.1 Relationship between anchor node ratio and normalized mean location error

As can be seen from Fig. 1, when the total number of nodes and the communication radius of nodes remain unchanged, the average positioning error and mean square deviation of the two algorithms decrease with the increase of the ratio of anchor nodes, and tend to be stable gradually. In addition, under the same conditions, the average positioning error of HADV-Hop is significantly smaller than that of DV-Hop, specifically, the normalized mean positioning error of HADV-Hop is reduced by 31\%-35\% compared with that of DV-Hop.

Fig. 2 is a graph of 15 anchor nodes with communication radius $\mathrm{R}=30 \mathrm{~m}$, the normalized average positioning error of the two algorithms varies with the total number of nodes. As can be seen from Figure 2, the positioning error and the mean square deviation of the two positioning algorithms decrease gradually with the increase of the number of anchor nodes, and tend to be stable. The positioning error of HADV-Hop is reduced by $29 \%-33 \%$ compared with that of DV-Hop.

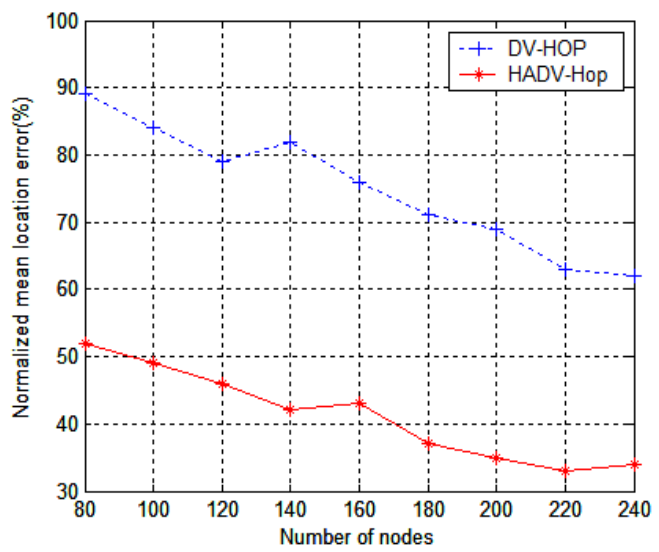

Fig. 2 Relationship between node number and normalized mean location error

From the above results, we can see that the proposed HADV-Hop algorithm is superior to the traditional DV-Hop algorithm in positioning accuracy, which shows that the combination of particle swarm algorithm and artificial bee colony algorithm after one iteration can effectively improve the 
positioning accuracy of wireless sensor network nodes; compared with the traditional DV-Hop algorithm, the positioning error is also higher. It is proved that the algorithm proposed in this paper has a great improvement in localization, and effectively improves the convergence speed of the artificial bee colony algorithm and improves the calculation accuracy of the algorithm.

\section{Conclusions}

Aiming at the problem that the search speed of artificial bee colony algorithm (DV-Hop) is slow and the precision is not high when calculating the unknown node coordinates, an improved artificial bee colony algorithm by mixing it with particle swarm optimization (PSO) called HADV-Hop is presented in this paper, HADV-Hop optimizes the calculation process of unknown node coordinates by improving DV-Hop algorithm without increasing hardware overhead, the positioning accuracy is improved and the positioning error is reduced. Simulation results show that the improved algorithm effectively improves the positioning accuracy and stability, and it is a simple and practical solution to the positioning problem of wireless sensor network nodes.

\section{References}

[1] KARABOGA D. An idea based on honey bee swarm for numerical optimization, Technical Report-TR06[R]. Kayseri: Erciyes University, Engineering Faculty, Computer Engineering Department, 2005.

[2] JIANG Tao. Improved Localization Algorithm of DV-Hop Based on Hybrid Artificial Bee Colony Algorithm [J]. Chinese Journal of Electron Devices, 2014, 37(5):912-916

[3] ZHAO Hongcai, ZHAO Xiaojie, WANG Maoli, et.al. DV-Hop improved algorithm based on fusion of differential evolution algorithm and artificial bee colony algorithm [J].Modern Electronics Technique, 2017, (15):129-132

[4] KARABOGA D, BASTURK B. On the performance of artificial bee colony (ABC) algorithm [J]. Applied Soft Computing, 2008, 8(1):687-697.

[5] LI Mu-dong, XIONG Wei, GUO Long. Improvement of DV-Hop Localization Based on Artificial Bee Colony Algorithm[J].Computer Science,2013,40(1):33-36. 